M. Yasumoto

Nagoya Math. J.

Vol. 70 (1978), 167-172

\title{
A NOTE ON SOME ORDERED RING
}

\author{
MASAHIRO YASUMOTO
}

An ordered ring with the least positive element 1 is a " $Z$-ring" if for each natural number $n$,

$$
\forall x \exists y \exists m(x=n y+m) \quad 0 \leqq m<n .
$$

An element $x \neq 0$ of a $Z$-ring is "infinitely divisible" if for infinitely many natural numbers $n$,

$$
\exists y(x=n y) \text {. }
$$

For example, $Z$ (the set of integers) is a $Z$-ring with no infinitely divisible element. Another example of $Z$-rings is $R=\{f(X) \in Q[X] \mid f(0)$ $\in Z$ \} where $\boldsymbol{Q}$ is the set of rationals and $X$ is placed greater than all rationals. Then $R$ has infinitely divisible elements, $X, X^{2}$, etc. In this paper we prove

THEOREM. There exists a Z-ring $A(\neq Z)$ which has no infinitely divisible element.

Remark 1. The ring $A$ which we construct has the following additional properties.

1) $\forall x \forall a>0 \quad \exists y \exists b(x=a y+b \& 0 \leqq b<a)$.

2) $A$ is a unique factorization domain, i.e. every element can be uniquely factorized to a finite product of prime elements.

The existence of such $Z$-ring was suggested by R. Kurata. (see Remark 2)

We introduce some notations. (refer to [1]). Let $N$ be the set of natural numbers. We say that $F \subset P(N)$ (the power set of $N$ ) is "a nonprincipal ultrafilter" if

1) $a \in F \& b \in F$ imply $a \cap b \in F$.

2) $a \in F \& a \subset b$ imply $b \in F$.

3) $a \notin F$ implies $N-a \in F$.

Received May 26, 1977. 
4) If $a$ is finite then $a \notin F$.

We introduce an equivalence relation by $F$ into $Z^{N}=Z \times Z \times \cdots$ as follows.

$$
\left(n_{0}, n_{1}, n_{2}, \cdots\right)_{\tilde{F}}\left(m_{0}, m_{1}, m_{2}, \cdots\right)
$$

if and only if

$$
\left\{i \in N \mid n_{i}=m_{i}\right\} \in F \text {. }
$$

Since $F$ is an ultrafilter, $\tilde{F}$ is the equivalence relation. We say that $Z^{N} / \tilde{F}$ is the ultrapower of $Z$ and denote it by $Z^{*}$. Let $\left(n_{i}\right)^{*}$ be the equivalence class of $\left(n_{i}\right)$. We can well define

$$
\begin{aligned}
& \left(n_{i}\right)^{*}+\left(m_{i}\right)^{*}=\left(n_{i}+m_{i}\right)^{*} \\
& \left(n_{i}\right)^{*} \cdot\left(m_{i}\right)^{*}=\left(n_{i} \cdot m_{i}\right)^{*} \\
& \left(n_{i}\right)^{*} \leqq\left(m_{i}\right)^{*} \quad \text { if }\left\{i \in N \mid n_{i} \leqq m_{i}\right\} \in F .
\end{aligned}
$$

We may assume $\boldsymbol{Z} \subset Z^{*}$ by identifying $n$ with $(n, n, n, \cdots)^{*}$.

By Los's theorem [1] $Z^{*}$ is the elementary extension of $Z$, in other words, for any first-order formula $\phi\left(v_{1}, v_{2}, \cdots, v_{k}\right)$ of the language of the ordered ring and for any integers $n_{1}, n_{2}, \cdots, n_{k}, \phi\left(n_{1}, n_{2}, \cdots, n_{k}\right)$ holds in $Z^{*}$, if and only if it holds in $Z$. For example, "the axioms of the ordered ring" and " $\forall x \forall a>0 \quad \exists y \exists b(x=a y+b), 0 \leqq b<a$ " are firstorder formulae. So $Z^{*}$ is a $Z$-ring. But "there is no infinitely divisible element" can not be a first-order formula. In fact, $Z^{*}$ has infinitely divisible elements, $\left(2,2^{2}, 2^{3}, \ldots\right)^{*},(1 !, 2 !, 3 !, 4 !, \ldots)^{*}$, etc.

In the following we construct a subring $A$ of $Z^{*}$ which satisfies the theorem.

Proof of the theorem. Let $p_{n}$ be the $n$-th prime number,

$$
A_{n, m}=\left\{k p_{n}^{m !}+\sum_{i=1}^{m-1} p_{n}^{i !}+\left[\log p_{n}\right] \mid k=0, \pm 1, \pm 2, \cdots\right\}
$$

where "[ ]" denotes the integer part.

Obviously, $m_{1} \leqq m_{2}$ implies $A_{n, m_{1}} \supset A_{n, m_{2}}$. Since $p_{1}^{n !}, p_{2}^{n !}, \cdots, p_{n}^{n !}$ are mutually prime, $B_{n}=\bigcap_{i=1}^{n} A_{i, n}$ is not empty. Pick $0 \leqq c_{n} \in B_{n}$ and define $c=\left(c_{1}, c_{2}, \cdots, c_{n}, \cdots\right)$.

Let $A^{\prime}=\left\{f\left(c^{*}\right) \in Z^{*} \mid f(X) \in Z[X]\right\}$ and

$$
A=\left\{z \in Z^{*} \mid \exists n \in Z\left(n \neq 0 \& n z \in A^{\prime}\right)\right\} .
$$

We prove that $A$ satisfies the theorem. 
By the definition of $A$ and by the fact that $Z^{*}$ is a $Z$-ring, it is easily checked that $A$ is a $Z$-ring. By the definition of $c, c^{*}$ is infinitely large in $Z^{*}$ i.e. for each $n \in Z\left(n<c^{*}\right)$ in $Z^{*}$. So $A \neq Z$.

For each $x \in A^{\prime}$, we define $f_{x}(X) \in Z[X]$ to be $f_{x}\left(c^{*}\right)=x$. We write $x \mid y$ if $\exists z(y=z x)$. We prove that there is no infinitely divisible element in $A$.

Lemma 1. For each $x \in A,\left\{n \in \boldsymbol{Z}\left|p_{n}\right| x\right.$ in $\left.\boldsymbol{Z}^{*}\right\}$ is finite.

Proof. We may assume $x \in A^{\prime}$.

By the definition of $c$,

$$
\begin{aligned}
c^{*} & \equiv\left[\log p_{n}\right] & & \left(\bmod p_{n}\right) \\
\left(c^{*}\right)^{k} & \equiv\left[\log p_{n}\right]^{k} & & \left(\bmod p_{n}\right) \\
x & \equiv f_{x}\left(\left[\log p_{n}\right]\right) & & \left(\bmod p_{n}\right) .
\end{aligned}
$$

Since $f_{x}(X) \in Z[X]$,

$$
\lim _{n \rightarrow \infty} \frac{f_{x}\left(\left[\log p_{n}\right]\right)}{p_{n}}=0 .
$$

Therefore, for all but finitely many $n$,

$$
\left|f_{x}\left(\left[\log p_{n}\right]\right)\right|<p_{n} .
$$

Since $\left\{n \in Z \mid f_{x}\left(\left[\log p_{n}\right]\right)=0\right\}$ is finite, for all but finitely many $n$,

$$
x \not \equiv \quad\left(\bmod p_{n}\right) \text {. }
$$

The result follows.

Lemma 2. For each $x \in A$ and each $n \in N$,

$$
\left\{m \in \boldsymbol{Z}\left|p_{n}^{m !}\right| x \text { in } \boldsymbol{Z}^{*}\right\} \quad \text { is finite. }
$$

Proof. Similar to the proof of Lemma 1. We may assume $x \in A^{\prime}$. By the definition of $c$,

$$
\begin{aligned}
c^{*} & \equiv \sum_{i=1}^{m-1} p_{n}^{i !}+\left[\log p_{n}\right] & \left(\bmod p_{n}^{m !}\right) \\
\left(c^{*}\right)^{k} & \equiv\left(\sum_{i=1}^{m-1} p_{n}^{i !}+\left[\log p_{n}\right]\right)^{k} & \left(\bmod p_{n}^{m !}\right) \\
x & \equiv f_{x}\left(\sum_{i=1}^{m-1} p_{n}^{i !}+\left[\log p_{n}\right]\right) & \left(\bmod p_{n}^{m !}\right) .
\end{aligned}
$$


Since $f_{x}(X) \in Z[X]$,

$$
\left|\lim _{m \rightarrow \infty} \frac{f_{x}\left(\sum_{i=1}^{m-1} p_{n}^{i !}+\left[\log p_{n}\right]\right)}{p_{n}^{m !}}\right| \leqq \lim _{m \rightarrow \infty} \frac{K p_{n}^{M \cdot(m-1) !}}{p_{n}^{m !}}=0
$$

where $K$ and $M$ are some constant numbers depending only on $f_{x}(X)$.

Therefore for all but finitely many $m$,

$$
\left|f_{x}\left(\sum_{i=1}^{m-1} p_{n}^{i !}+\left[\log p_{n}\right]\right)\right|<p_{n}^{m !} .
$$

Since $\left\{m \in Z \mid f_{x}\left(\sum_{i=1}^{m-1} p_{n}^{i !}+\left[\log p_{n}\right]\right)=0\right\}$ is finite, for all but finitely many $m$,

$$
x=f_{x}\left(\sum_{i=1}^{m-1} p_{n}^{i !}+\left[\log p_{n}\right]\right) \quad\left(\bmod p_{n}^{m !}\right)
$$

and

$$
0<\left|f_{x}\left(\sum_{i=1}^{m-1} p_{n}^{i !}+\left[\log p_{n}\right]\right)\right|<p_{n}^{m !}
$$

This proves Lemma 2.

By lemma 1 and lemma 2, every $x \in A$ is not infinitely divisible in $Z^{*}$, and therefore so is in $A$. So our theorem is proved.

Remark. Our original motivation is to construct a model which resembles the set of natural numbers, but is not the same. The positive part of $A$ above constructed resembles the set of natural numbers in the following sence. (It is easily checked.)

1) The positive part of $A$ satisfies mathematical induction for any formula $\phi(x)$ of the language $L=\langle+,=,\langle\rangle$.

2) The positive part of $A$ satisfies mathematical induction of the product form. Namely, for any formula $\phi(x)$ of the language $L=$ $\langle+,=, \cdot,\langle\rangle$, if $\phi(1), \phi(p)$ for any prime $p$, and

$$
\forall x<a(x \mid a \rightarrow \phi(x)) \rightarrow \phi(a), \quad \text { then } \forall x \phi(x) .
$$

On the other hand, the theorem of Lagrange does not hold. For example, $c^{*}$ can not be a sum of squares.

Further results about $A$ above constructed.

In the following, we prove that $A$ cannot be an Euclidean ring (Lemma 3), but admits Euclidean algorithm (Lemma 4).

Let $a$ and $b$ be elements of $A$. We define $a \leqslant b$ iff $b-a>n$ for any $n \in \boldsymbol{Z}$. 
LEMMA 3. A cannot be an Euclidean ring.

Proof. If not, there exist a well-ordered set $W$ and a map $\rho$ from $A$ onto $W$ such that

(*) $\forall x \forall a \exists y \exists b \quad x=a y+b$ and $\rho(b)<\rho(a)$.

Let $B=\{\rho(x) \mid x \in A-Z\}$. Then there is an element $a_{0} \in A-Z$ such that $\rho\left(a_{0}\right)$ is the least element of $B$. We may assume that $a_{0}>0$. We take an $x_{0}$ such that $0 \ll x_{0} \ll a_{0}$.

By $(*)$, there exist $y$ and $b$ such that

$$
x_{0}=a_{0} y+b \text { and } \rho(b)<\rho\left(a_{0}\right) .
$$

Then by the definition of $a_{0}, b \in \boldsymbol{Z}$.

Since 1 is the least positive element, $y \geqq 1$. So $x_{0}-b \geqq a_{0}$. This is contrary to $x_{0} \ll a_{0}$.

Let $a$ be an element of $A$, then there exist $f(X) \in Z[X]$ and $n \in Z$ such that $a=f\left(c^{*}\right) / n$. We can well define $\operatorname{deg}(a)=\operatorname{deg}(f(X))$.

We notice that $a<b$ implies $\operatorname{deg}(a) \leqq \operatorname{deg}(b)$.

LEMMA 4. A admits Euclidean algorithm.

Proof. Let $a$ and $b$ be elements of $A$ and assume $a>b>0$.

We prove by induction on $\operatorname{deg}(a)$.

(1) If $\operatorname{deg}(a)=0$, then $a, b \in Z$. This case is obvious.

(2a) Let $\operatorname{deg}(a)=n$ and $\operatorname{deg}(b)<n$.

There exist $y$ and $d$ such that

$$
a=b y+d \text { and } 0 \leqq d<b .
$$

Then $\operatorname{deg}(d) \leqq \operatorname{deg}(b)<n$. By the induction hypothesis, Euclidean algorithm for $b$ and $d$ exists.

(2b) Let $\operatorname{deg}(a)=\operatorname{deg}(b)=n$.

We can write

$$
\begin{aligned}
& a=\frac{1}{m}\left(a_{0} c^{* n}+\cdots+a_{n}\right) \\
& b=\frac{1}{m}\left(b_{0} c^{* n}+\cdots+b_{n}\right)
\end{aligned}
$$

where $m, a_{0}, \cdots, a_{n}, b_{0}, \cdots, b_{n}$ are elements of $Z$ and $0<b_{0} \leqq a_{0}$. 
Since $a_{0}, b_{0} \in Z$, there is a system of equations

$$
\begin{array}{ll}
a_{0}=q_{1} b_{0}+r_{1} & \\
b_{0}=q_{2} r_{1}+r_{2} & \vdots \\
r_{k}=q_{k+2} r_{k+1} & \left(\begin{array}{l}
q_{1}, q_{2}, \ldots, q_{k+2}, r_{1}, r_{2}, \ldots, r_{k+1} \in Z \\
b_{0}>r_{1}>r_{2}>\ldots>r_{k+1}>0
\end{array}\right)
\end{array}
$$

Then

$$
\begin{array}{rlr}
a & =q_{1} b+R_{1} \\
b & =q_{2} R_{1}+R_{2} & \\
R_{k} & =q_{k+2} R_{k+1}+R_{k+2}
\end{array} \quad\left(\begin{array}{l}
\text { If } 1 \leqq i \leqq k+1, \\
R_{i}=\frac{1}{m}\left(r_{i} c^{* n}+\cdots\right) . \\
\operatorname{deg}\left(R_{k+2}\right)<n .
\end{array}\right)
$$

So case $(2 b)$ is reduced to $(2 a)$.

\section{REFERENCES}

[1] Bell, J. L. and Slomson, A. B.: Models and Ultraproducts. Amsterdam, NorthHolland Publishing Company, 1969.

[2] Chang, C. C. and Keisler, J.: Model Theory, North-Holland.

Nagoya University 